\section{Neurología hospitalaria y COVID-19: serie de 96 pacientes evaluados en un hospital universitario}

\author{
JAIME GODOY-SANTÍN ${ }^{1, a}$, SEBASTIÁN BRAVO GRAU², \\ FELIPE NUÑEZ ${ }^{\mathrm{b}}$, CECILIA AGUILAR ${ }^{1}$, DIEGO GUTIÉRREZ1, \\ HÉCTOR MIRANDA ${ }^{1}$, PATRICIO SANDOVAL RUBIO ${ }^{1}$, \\ BERNARDITA RAMOS ${ }^{c}$, LORENA GARCÍA A. ${ }^{c}$, \\ MAX ANDRESEN ${ }^{3}$, PATRICIO MELLADO ${ }^{1}$
}

\section{Neurology and COVID-19: Case Series of Neurological Complications in 96 patients Admitted at a University Hospital}

Background: There are multisystemic consequences secondary to SARSCoV-2 infection. Aim: To characterize neurological complications in patients admitted due to SARS-CoV-2 infection. Methods: Review of medical records of patients aged over 15 years with COVID-19 evaluated by the neurology team between April and August 2020 at a university hospital. Severity of the infection, referral reasons, neurological diagnoses and laboratory results were registered. The diagnoses were defined by consensus among the members of the hospital neurology group. Cerebrovascular and inflammatory diseases of the central and peripheral nervous system were defined as "probably associated" or "possibly associated" to COVID-19. Results: Ninety-six patients had at least 1 new neurological complication. $74 \%$ were admitted due to pneumonia and $20 \%$ due to a neurological disease. The most common reasons for neurological referral were impaired consciousness (39\%), focal neurological deficit (24\%), headache (9\%) and seizures (5\%). The most relevant neurological diagnoses were delirium in 48 patients, stroke in 24, critical illness polyneuropathy and myopathy in 17, seizures in 14, brachial plexopathy in 3, compressive neuropathies in 5, encephalitis in 1, possible vasculitis in 1 and Guillain-Barré syndrome in 1. Stroke and epilepsy were associated with increased length of hospital stay, but without differences in mortality. Conclusions: The spectrum of neurological complications of COVID-19 is wide. There are clinical entities typical of critically ill patients and also diseases associated directly and indirectly with the SARS-CoV2 infection.

(Rev Med Chile 2021; 149: 527-532)

Key words: COVID-19; Delirium; Neurology; Seizures; Stroke.
'Departamento de Neurología, Escuela de Medicina, Pontificia Universidad Católica de Chile. Santiago, Chile.

${ }^{2}$ Departamento de Radiología, Escuela de Medicina, Pontificia Universidad Católica de Chile.

Santiago, Chile.

${ }^{3}$ Departamento de Medicina Intensiva, Escuela de Medicina, Pontificia Universidad Católica de Chile. Santiago, Chile.

${ }^{a}$ Grupo de trabajo AIRR-UC, Facultad de Medicina, Pontificia Universidad Católica de Chile.

Santiago, Chile.

bInterno, Escuela de Medicina,

Pontificia Universidad Católica de

Chile. Santiago, Chile.

'Residente de Neurología,

Escuela de Medicina, Pontificia

Universidad Católica de Chile.

Santiago, Chile.

Financiamiento: Trabajo no recibió financiamiento.

Los autores declaran no tener conflictos de interés.

Recibido el 14 de octubre de 2020, aceptado el 19 de enero de 2021.

Correspondencia a:

Patricio Mellado Talesnik Departamento de Neurología. Diagonal Paraguay $362,5^{\circ}$ piso. Santiago, Chile.

pmelladotalesnik@gmail.com
A fines de 2019 se comunicaron los primeros casos de neumonías por un nuevo betacoronavirus que posteriormente se denominó SARS-CoV-2 ${ }^{1}$. Tras su identificación se produjo una rápida expansión, generando una pandemia que afecta a Chile desde marzo de 2020. La enfermedad producida por el virus, denominada COVID-19, se caracteriza principalmente por fiebre, compromiso del estado general y síntomas respiratorios ${ }^{2}$. Más de un tercio de los pacientes hospitalizados con COVID-19 desarrolla síntomas neurológicos, siendo los más frecuentes cefalea, anosmia/hiposmia, hipogeusia/disgeusia y mareos ${ }^{3}$. Sin embargo, también están descritas 
complicaciones neurológicas graves, destacando enfermedad cerebrovascular ${ }^{4}$, crisis epilépticas ${ }^{5}$, meningoencefalitis ${ }^{6}$, síndrome de Guillain-Barré $^{7,8}$, delirium ${ }^{3,9}$ y mioneuropatía, entre otras. Algunas de estas complicaciones son las propias del paciente crítico, mientras que en otras la misma infección podría tener un rol específico. La fisiopatología no es del todo clara, pero la evidencia acumulada hasta la fecha sugiere que la presencia del receptor de enzima convertidora de angiotensina 2 (ACE2) en el sistema nervioso central podría favorecer una invasión directa ${ }^{10}$. Los trastornos neurológicos podrían explicarse también por complicaciones indirectas de la enfermedad, tales como la respuesta inflamatoria sistémica, la disfunción de órganos y el estado protrombótico ${ }^{9,11}$. Hasta la fecha no hay publicaciones en nuestro medio que describan qué proporción de pacientes hospitalizados por COVID-19 requieren evaluación neurológica, cuáles son los motivos de evaluación, ni cuál es la frecuencia de complicaciones neurológicas. Los objetivos de nuestro trabajo son describir las complicaciones neurológicas más frecuentes en pacientes hospitalizados con diagnóstico de COVID-19 y buscar la relación entre éstas y gravedad de COVID-19, estadía hospitalaria y mortalidad.

\section{Material y Método}

Se incluyeron todos los casos mayores de 15 años de COVID-19 confirmados por laboratorio de acuerdo a las definiciones establecidas por la Organización Mundial de la Salud ${ }^{12,13}$ que requirieron evaluación por el equipo de neurología hospitalaria durante su estadía en el Hospital Clínico de la Red UC-CHRISTUS; se excluyeron a los pacientes cuyos diagnósticos neurológicos eran conocidos previo a la infección por COVID-19. Al no existir estudios de prevalencia de complicaciones neurológicas en pacientes con COVID-19 en nuestra población se decidió realizar un muestreo por conveniencia de pacientes evaluados entre el 3 de abril y el 7 de agosto de 2020. Se revisaron fichas clínicas, laboratorio, estudios electrofisiológicos y neuroimágenes de todos los pacientes. Se registró edad, sexo, fecha de inicio de síntomas de COVID-19, motivo de consulta, fecha de inicio de síntomas neurológicos, exámenes de laboratorio (dímero $\mathrm{D}, \mathrm{LDH}$, ferritina, fibrinógeno, IL-6, estudio de líquido céfalo-raquídeo), hallazgos de neuroimágenes, electroencefalogramas, estadía hospitalaria y condición de egreso. Se consideró como día de inicio del COVID-19 el primer día de síntomas respiratorios. La gravedad del COVID-19 fue clasificada de acuerdo a lo reportado por Wu y McGoogan ${ }^{14}$. Todos los pacientes fueron evaluados por neurólogos hospitalistas (JG, PM, CA, PS, HM, DG). El diagnóstico neurológico fue establecido por consenso por los miembros del equipo. Las imágenes fueron revisadas por neurorradiólogo (SB). Las enfermedades cerebrovasculares y los trastornos inflamatorios centrales y periféricos fueron catalogados como "probablemente asociados a COVID-19" y "posiblemente asociados a COVID-19" de acuerdo a los criterios establecidos por Ellul y colegas ${ }^{15}$.

Para la estadística descriptiva se utilizó media y desviación estándar para las variables continuas, y número y porcentaje para las categóricas. Se efectuó un análisis descriptivo, con prueba de Chi-cuadrado para las variables dicotómicas y t de Student para comparar variables cuantitativas. Se consideró estadísticamente significativo $\mathrm{p}<0,05$.

El estudio fue aprobado por el Comité de Ética Científico en Ciencias de la Salud de la Pontificia Universidad Católica de Chile (número 200708007).

\section{Resultados}

Durante el período del estudio el Hospital Clínico UC-CHRISTUS registró un total de 1.064 pacientes con COVID-19 confirmado, de los cuales 120 requirieron evaluación por el equipo de Neurología. De ellos, 24 fueron excluidos del análisis por presentar diagnósticos neurológicos conocidos; 71 de los 96 pacientes analizados (74\%) ingresaron por neumonía COVID-19, 19 (20\%) por enfermedad neurológica y $6(6 \%)$ por otras manifestaciones; 42 casos (44\%) fueron catalogados como COVID-19 crítico, 31 como COVID-19 grave $(32 \%)$ y $23(24 \%)$ como leves y 9 fueron asintomáticos (Tabla 1).

Los motivos de evaluación neurológica más frecuentes fueron compromiso de conciencia (39\%), déficit neurológico focal $(24 \%)$, cefalea (9\%), crisis epilépticas (5\%) y movimientos involuntarios (3\%) (Figura 1). Setenta y ocho pacientes fueron estudiados con neuroimágenes 
Tabla 1. Características demográficas de pacientes con COVID-19 con complicaciones neurológicas

\begin{tabular}{|c|c|c|c|c|}
\hline & $\begin{array}{c}\text { Total } \\
(n=96)\end{array}$ & $\begin{array}{l}\text { COVID-19 } \\
\text { leve } \\
(n=23)\end{array}$ & $\begin{array}{l}\text { COVID-19 } \\
\text { grave } \\
(n=31)\end{array}$ & $\begin{array}{l}\text { COVID-19 } \\
\text { crítico } \\
(n=42)\end{array}$ \\
\hline Sexo masculino (n, \%) & $58(60)$ & $12(52,2)$ & $12(38,7)$ & $34(81)$ \\
\hline Edad (promedio, DE) & $60,5( \pm 17,6)$ & $59,7( \pm 21,4)$ & $63,6( \pm 19,4)$ & $58,8( \pm 13,8)$ \\
\hline $\begin{array}{l}\text { Comorbilidades (n, \%) } \\
\text { HTA } \\
\text { DM } \\
\text { ERC } \\
\text { Enfermedad vascular previa (ACV, CC, EAP) } \\
\text { Cáncer activo }\end{array}$ & $\begin{array}{l}53(55) \\
27(28) \\
12(13) \\
12(13) \\
11(11)\end{array}$ & $\begin{array}{c}10(43,5) \\
5(21,7) \\
3(13) \\
4(17.4) \\
1(4,3)\end{array}$ & $\begin{array}{r}17(54,8) \\
11(35,5) \\
4(12,9) \\
4(12,9) \\
6(19,4)\end{array}$ & $\begin{array}{r}26(61,9) \\
11(26,2) \\
5(11,9) \\
4(9,5) \\
4(9,5)\end{array}$ \\
\hline $\begin{array}{l}\text { Motivo ingreso } \\
\text { Neumonía COVID-19 } \\
\text { Enfermedad neurológica } \\
\text { Otro }\end{array}$ & $\begin{array}{r}71(74) \\
19(20) \\
6(6)\end{array}$ & $\begin{aligned} 5 & (21,7) \\
15 & (65,2) \\
3 & (13)\end{aligned}$ & $\begin{array}{r}25(80,6) \\
4(12,9) \\
2(6,5)\end{array}$ & $\begin{array}{c}40(95,2) \\
0 \\
2(4,8)\end{array}$ \\
\hline
\end{tabular}

DE: desviación estándar; HTA: hipertensión arterial crónica; DM: diabetes mellitus; ERC: enfermedad renal crónica; ACV: accidente cerebrovascular; CC: cardiopatía coronaria; EAP: enfermedad arterial periférica.

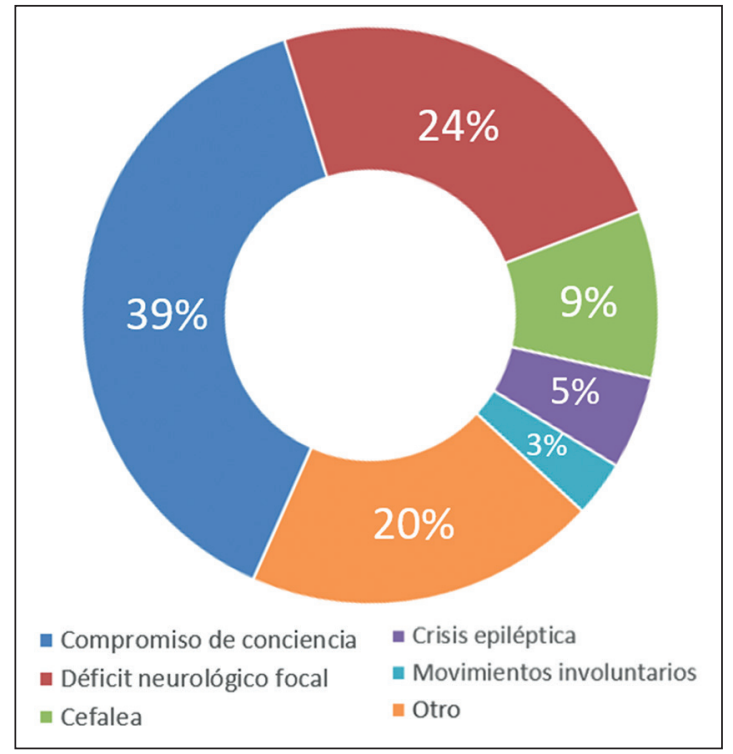

Figura 1. Motivos de evaluación neurológica.

(50 con tomografía computada y 28 con resonancia magnética), 52 con electroencefalograma y 18 con punción lumbar. Dentro de los diagnósticos realizados destacaron 24 casos de accidente cerebrovascular (ACV) (17 infartos, 3 de hemorragias intraparenquimatosas, 1 de hemorragia subaracnoidea, 3 casos de trombosis venosas encefálicas), 1 de posible vasculitis, 1 caso de síndrome de hiperperfusión cerebral, 4 de encefalopatías post-paro, 14 de crisis epilépticas, 1 síndrome de Guillain-Barré, 5 neuropatías periféricas, 3 de plexopatías braquiales y 5 casos de cefaleas primarias o posiblemente asociadas a COVID-19 (Tabla 2). En 48 casos se diagnosticó delirium y en 17 mioneuropatía de paciente crítico.

\section{Accidente cerebrovascular}

Las principales características de los subgrupos de ACV se describen en la Tabla 3. Diecisiete pacientes ( 12 hombres y 5 mujeres) presentaron infartos encefálicos, 11 de ellos durante la estadía hospitalaria. Los antecedentes más frecuentes fueron hipertensión arterial crónica $(65 \%)$, diabetes mellitus tipo 2 (29\%), eventos vasculares previos $(24 \%)$ y cáncer $(24 \%)$. Todos los infartos encefálicos fueron catalogados como embólicos y en 2 (12\%) de ellos las imágenes mostraron oclusión de un gran vaso intracraneano. Seis casos $(35 \%)$ fueron de origen cardioembólico y 4 (24\%) por enfermedad de grandes vasos. Dos de los casos cumplieron criterios de asociación probable a COVID-19. En 6 casos (35\%) se encontraron eventos trombóticos concomitantes en otros territorios (4 tromboembolismos pulmonares, 1 infarto agudo al miocardio, 1 isquemia extremidades inferiores).

Tres pacientes (2 hombres y 1 mujer, 55-89 años) presentaron hemorragias intraparenquima- 
Tabla 2. Complicaciones neurológicas seleccionadas en pacientes con COVID-19

\begin{tabular}{|lcccc|}
\hline & $\begin{array}{c}\text { Total } \\
(\mathbf{n}=\mathbf{9 6})\end{array}$ & $\begin{array}{c}\text { CoviD-19 } \\
\text { leve }(\mathbf{n}=\mathbf{2 3})\end{array}$ & $\begin{array}{c}\text { CoviD-19 } \\
\text { grave }(\mathbf{n}=\mathbf{3 1})\end{array}$ & $\begin{array}{c}\text { Covid-19 } \\
\text { crítico (n= 42) }\end{array}$ \\
Accidente cerebrovascular & 24 & 13 & 3 & 8 \\
Crisis epilépticas & 14 & 3 & 4 & 7 \\
Plexopatías y neuropatías compresivas & 8 & 0 & 1 & 7 \\
\hline
\end{tabular}

tosas, todas en sitios típicamente hipertensivos. En 1 de los casos no se encontró causas para la hemorragia y se cumplieron criterios de asociación probable a COVID-19. Una paciente de 43 años previamente sana ingresó por hemorragia subaracnoidea secundaria a un aneurisma roto de arteria comunicante posterior de $3,4 \times 2,4 \mathrm{~mm}$, con criterios de asociación probable a COVID-19.

Se evaluaron 3 pacientes previamente sanos ( 2 mujeres, 1 hombre, 26-46 años) con trombosis venosas encefálicas, 2 en senos venosos y 1 en vena cortical en contexto de hipovolemia intracraneana. En 1 de los casos de trombosis de seno venoso no se encontraron causas ni factores de riesgo.

Al comparar los grupos de pacientes con y sin ACV no se encontró asociación significativa con niveles de parámetros inflamatorios (PCR, IL-6, ferritina) ni dímero D. Se encontró una asociación no significativa entre ACV y COVID-19 leve que persistió tras corregir por sexo, edad, HTA, DM y enfermedad vascular previa. La mediana de estadía hospitalaria fue mayor en comparación a la población total de pacientes con COVID-19 ( 18 vs 9 días, $\mathrm{p}=0,005$ ) pero no hubo diferencias significativas en mortalidad hospitalaria $(16,7 \%$ vs $12 \%, \mathrm{p}=0,481)$.

\section{Crisis epilépticas}

En 14 pacientes (42,9\% hombres, edad 61,9 \pm $21,1)$ se diagnosticaron crisis epilépticas. Cuatro pacientes debutaron como estado epiléptico, 3 de ellos no convulsivos. En relación a la etiología, 7 crisis se presentaron en relación a trastornos tóxico-metabólicos, 4 en contexto de enfermedad cerebrovascular (trombosis venosa encefálica, síndrome de hiperperfusión e infartos múltiples, vasculitis), 1 por encefalitis, 1 en contexto de encefalopatía hipóxico-isquémica y 1 en relación a un trastorno neurodegenerativo. No se encontró relación entre crisis epilépticas y gravedad de COVID-19. La mediana de estadía hospitalaria fue significativamente mayor en comparación a la población total de pacientes hospitalizados $(23$ vs 9 días, $\mathrm{p}=0,001)$. Al corregir por sexo y edad no hubo diferencias en mortalidad hospitalaria.

\section{Otros}

Se diagnosticó plexopatía braquial en 3 pacientes y mononeuropatías compresivas en 5 pacientes, todos con antecedente de haber recibido ventilación en prono. Una paciente ingresó por síndrome de Guillain-Barré que inició a los 7 días del COVID-19 y cumplió criterios de probable asociación. El estudio electrofisiológico resultó compatible con una polirradiculopatía desmielinizante.

Destacaron además otros 2 síndromes inflamatorios: una encefalitis asociada a COVID-19 y una posible vasculitis cerebral asociada a COVID-19.

En 5 casos el diagnóstico principal fue cefalea primaria o posiblemente asociada a COVID-19; todos fueron estudiados con neuroimágenes que no mostraron hallazgos de carácter agudo y en 2 casos se realizó una punción lumbar que mostró análisis citoquímicos normales.

\section{Discusión}

El 11,2\% de los pacientes hospitalizados con COVID-19 requirió evaluación por el equipo de neurología hospitalaria y en $80 \%$ de estos se constató un nuevo trastorno neurológico. Aparte del delirium y la mioneuropatía del paciente crítico, las complicaciones neurológicas más frecuentes fueron ACV $(2,3 \%)$ y crisis epilépticas $(1,3 \%)$, cifras similares a lo reportado en la literatura ${ }^{3,15,16}$. Destacaron además trastornos inflamatorios (1 posible vasculitis, 1 encefalitis, 1 síndrome de Guillain-Barré), mononeuropatías compresivas ( 5 casos) y plexopatías braquiales (3 casos).

Se han postulado varios mecanismos fisiopato- 
lógicos para explicar la relación entre COVID-19 y ACV. Si bien es reconocido que cualquier proceso infeccioso aumenta el riesgo de $\mathrm{ACV}$, se postula que en el caso del SARS-CoV-2 existen mecanismos específicos que podrían predisponer a infartos y hemorragias intracraneanas. El estado protrombótico que caracteriza al COVID-19 y el aumento de la viscosidad podrían favorecer los eventos isquémicos, mientras que la coagulopatía de consumo que se observa en algunos casos aumentaría el riesgo de hemorragias ${ }^{17,18}$. A estos procesos sistémicos se suma también el daño directo que se puede provocar a nivel miocárdico y endotelial, lo que favorecería arritmias, formación de trombos intracavitarios, microtrombosis de arterias penetrantes y hemorragias intracraneanas espontáneas ${ }^{18,19}$. Así, es esperable que el COVID-19 se asocie a infartos embólicos y de pequeños vasos. Si bien series iniciales sugerían asociación a oclusión de grandes vasos intracraneanos ${ }^{4}$ estudios posteriores mostraron que podía observarse todo tipo de infartos, incluyendo infartos lacunares ${ }^{19}$. En nuestra serie no encontramos infartos de pequeño vaso, lo que podría explicarse por el bajo tamaño muestral y la menor frecuencia de enfermedad lacunar en nuestro centro $(9,6 \%$ del total de los infartos entre 2018 y 2020, datos no publicados). En 76\% de los casos se logró establecer etiología (cardioembolia, enfermedad de grandes vasos y trombofilias adquiridas). Dentro del grupo criptogénico, en la mitad de los casos (12\%) no se encontró ningún factor de riesgo para enfermedad cerebrovascular, cumpliendo criterios de probable asociación a COVID-19; en ambos casos el COVID-19 fue crítico. En el caso de las hemorragias intraparenquimatosas y las trombosis venosas encefálicas, en 33\% de los casos no se encontraron factores de riesgo para enfermedad cerebrovascular, cumpliendo criterios de probable asociación a COVID-19.

Al comparar los grupos de pacientes con y sin ACV no encontramos diferencias significativas en los niveles de los parámetros inflamatorios ni dímero $\mathrm{D}$ y no hubo asociación a gravedad de COVID-19; de hecho, hubo una tendencia no significativa a asociación entre ACV y COVID-19 leve. Algunos factores que podrían explicar este resultado son la naturaleza retrospectiva de nuestro estudio, la búsqueda activa del virus que realizamos en nuestros pacientes y que la clasificación de la gravedad del COVID-19 se basa principalmente en manifestaciones pulmonares, sin considerar que el COVID-19 podría debutar con presentaciones extrapulmonares. Otra posible explicación es que en la mayoría de los casos la interconsulta al equipo de neurología y la solicitud de neuroimágenes se basó en las alteraciones encontradas en el examen físico, limitado por la condición clínica y el uso de fármacos, de modo que podría haber un subdiagnóstico en los pacientes con COVID-19 crítico. Finalmente, es posible que el COVID-19 no haya tenido un rol causal directo y que haya actuado más bien como un precipitante, lo que es concordante con que en la mayoría de nuestros casos se encontró una etiología establecida y que no hubo asociación con los niveles de los parámetros inflamatorios ni dímero D. Se necesitan de estudios prospectivos para aclarar la forma de asociación entre COVID-19 y ACV.

En nuestra serie, la mitad de las crisis epilépticas se observaron en pacientes con trastornos tóxico-metabólicos severos y el resto se dio principalmente en pacientes con enfermedad cerebrovascular. Sin embargo, también encontramos 2 casos con trastornos inflamatorios subyacentes (1 vasculitis, 1 encefalitis), por lo que creemos que en ausencia de causas claras se debe realizar estudio con RM y PL.

Finalmente cabe destacar que en 3 pacientes se diagnosticó plexopatía braquial; todos los casos se vieron en pacientes con COVID-19 crítico que requirieron ventilación en prono. Creemos que lo más probable es que estos casos se traten de plexopatías compresivas secundarias al decúbito en prono, lo que ha sido previamente descrito en pacientes sin COVID-1921. Un fenómeno similar podría explicar los 5 casos de mononeuropatías que encontramos.

La principal fortaleza de nuestro trabajo es que la mayoría de los pacientes fueron estudiados en forma exhaustiva, incluyendo resonancia magnética y estudios electrofisiológicos. Sin embargo, nuestro estudio también presenta limitaciones. Si bien los diagnósticos neurológicos fueron establecidos en la medida que nuestro equipo tomó contacto con los pacientes, debido a la naturaleza retrospectiva del trabajo podría haber sesgos en la adjudicación de otras variables. Por otra parte, el delirium y la mioneuropatía del paciente crítico son frecuentemente reconocidos y tratados por los médicos que trabajan en las unidades críticas y de complejidad intermedia y la solicitud de evalua- 
ción por neurólogos queda a juicio del tratante, por lo que no fue sistemática. Así, es altamente probable que en nuestro trabajo se hayan subestimado estas complicaciones. Finalmente, algunos de los pacientes se mantenían hospitalizados al momento del análisis, por lo que las complicaciones neurológicas y la mortalidad intrahospitalaria podrían estar subestimadas.

Como conclusión, el espectro de complicaciones neurológicas es amplio e incluye enfermedades potencialmente graves que pueden determinar mayor discapacidad y prolongar estadías. Algunos de estos trastornos son los propios del paciente crítico y otros podrían ser específicos de la infección por SARS-CoV-2. Estas complicaciones no son exclusivas de pacientes con COVID-19 grave o crítico, por lo que se debe mantener un bajo umbral para sospecharlas, incluso en pacientes con COVID-19 leve. Se espera que en el futuro próximo se desarrollen estudios prospectivos con seguimiento más prolongado para responder mejor cómo se asocian las distintas complicaciones neurológicas con COVID-19 y sus consecuencias de largo plazo.

\section{Referencias}

1. Ghinai I, McPherson TD, Hunter JC, Kirking HL, Christiansen D, Joshi K, et al. First known person-to-person transmission of severe acute respiratory syndrome coronavirus 2 (SARS-CoV-2) in the USA. Lancet 2020; 395 (10230): 1137-44.

2. Gandhi RT, Lynch JB, del Rio C. Mild or Moderate Covid-19. N Engl J Med 2020; 24: 1-9.

3. Mao L, Jin H, Wang M, Hu Y, Chen S, He Q, et al. Neurologic Manifestations of Hospitalized Patients with Coronavirus Disease 2019 in Wuhan, China. JAMA Neurol 2020; 77 (6): 683-90.

4. Oxley TJ, Mocco J, Majidi S, Kellner CP, Shoirah H, Singh IP, et al. Large-Vessel Stroke as a Presenting Feature of COVID-19 in the Young. N Engl J Med 2020; 382 (20): e60.

5. Asadi-Pooya AA. Seizures associated with coronavirus infections. Seizure 2020; 79: 49-52.

6. Moriguchi T, Harii N, Goto J, Harada D, Sugawara H, Takamino J, et al. A first case of meningitis/encephalitis associated with SARS-Coronavirus-2. Int J Infect Dis 2020; 94: 55-8.

7. Alberti P, Beretta S, Piatti M, Karantzoulis A, Piatti ML, Santoro P, et al. Guillain-Barré syndrome related to
COVID-19 infection. Neurol - Neuroimmunol Neuroinflammation 2020; 7 (4): e741.

8. Toscano G, Palmerini F, Ravaglia S, Ruiz L, Invernizzi P, Cuzzoni MG, et al. Guillain-Barré Syndrome Associated with SARS-CoV-2. N Engl J Med 2020; 17: 1-3.

9. Kotfis K, Williams Roberson S, Wilson JE, Dabrowski W, Pun BT, Ely EW. COVID-19: ICU delirium management during SARS-CoV-2 pandemic. Crit Care 2020; 24 (1): 1-9.

10. Zubair AS, McAlpine LS, Gardin T, Farhadian S, Kuruvilla DE, Spudich S. Neuropathogenesis and Neurologic Manifestations of the Coronaviruses in the Age of Coronavirus Disease 2019: A Review. JAMA Neurol 2020; 77 (8): 1018-27.

11. Klok FA, Kruip MJHA, van der Meer NJM, Arbous MS, Gommers DAMPJ, Kant KM, et al. Incidence of thrombotic complications in critically ill ICU patients with COVID-19. Thromb Res 2020; 191: 145-7.

12. World Health Organization. Coronavirus disease 2019 (COVID-19): situation report, 61. Geneva: World Health Organization 2020.

13. World Health Organization. Laboratory testing for 2019 novel coronavirus (2019-nCoV) in suspected human cases. Geneva: World Health Organization 2020.

14. Wu Z, McGoogan J. Characteristics of and important lessons from the coronavirus disease 2019(COVID-19) outbreak in China. JAMA 2020; 323 (13): 1239-42.

15. Ellul MA, Benjamin L, Singh B, Lant S, Michael BD, Easton A, et al. Neurological associations of COVID-19. Lancet Neurol 2020; 19 (9): 767-83.

16. Shahjouei S, Li SNJ, Khan A, Chaudhary D, Farahmand G, Male S, et al. Risk of Stroke in Hospitalized SARSCoV-2 Infected Patients: A Multinational Study. EBioMedicine 2020; 59: 1029393.

17. Beyrouti R, Adams ME, Benjamin L, Cohen H, Farmer SF, Goh YY, et al. Characteristics of ischaemic stroke associated with COVID-19. J Neurol Neurosurg Psychiatry 2020; 91 (8): 889-91.

18. Valderrama EV, Humbert K, Lord A, Frontera J, Yaghi S. Severe Acute Respiratory Syndrome Coronavirus 2 Infection and Ischemic Stroke. Stroke 2020; 51 (7): e124-7.

19. Ntaios G, Michel P, Georgiopoulos G, Guo Y, Li W, Xiong J, et al. Characteristics and Outcomes in Patients With COVID-19 and Acute Ischemic Stroke: The Global COVID-19 Stroke Registry. Stroke 2020; 13: 1-5.

20. Silveira DC, Sagi A, Romero R. Are seizures predictors of mortality in critically ill patients in the intensive care unit (ICU)? Seizure 2019; 73: 14-1.

21. Goettler CE, Pryor JP, Reilly PM. Brachial plexopathy after prone positioning. Crit Care. 2002; 6 (6): 540-2. 